\title{
EDITORIAL
}

\section{NK4 therapy: a new approach to target angiogenesis and inflammation in rheumatoid arthritis}

\author{
Bradley J Rabquer ${ }^{1,2}$ and Alisa E Koch ${ }^{2 *}$ \\ See related research by Tsunemi et al.: http://arthritis-research.com/content/15/4/R75
}

\begin{abstract}
Rheumatoid arthritis (RA) is a progressive autoimmune disease characterized by synovial membrane hyperplasia, inflammation, and angiogenesis. Hepatocyte growth factor (HGF) and its receptor, c-Met, are both overexpressed in the RA synovium. NK4 is an antagonist of HGF which has been shown to inhibit tumor growth, metastasis, and angiogenesis. In an experimental model of RA, NK4 gene therapy inhibited joint damage and inflammation in both preventative and therapeutic models. NK4 treatment therefore represents a possible therapeutic option in combating RA.
\end{abstract}

Rheumatoid arthritis (RA) is a progressive, inflammatory autoimmune disease characterized by an erosive synovitis. In addition to being an inflammatory condition, $\mathrm{RA}$ is also considered to be a member of the angiogenic family of diseases. Angiogenesis is growth of new blood vessels from pre-existing blood vessels. As the disease progresses, the hyperplastic synovial pannus creates a hypoxic, inflammatory environment that induces angiogenesis. Further vascularization of the synovial tissue promotes pannus growth and continued infiltration of inflammatory leukocytes, thus perpetuating the disease.

In the previous issue of Arthritis Research \& Therapy, Tsunemi and colleagues [1] reported on the targeting of hepatocyte growth factor (HGF) by NK4 in the treatment of RA. HGF is a pleiotropic growth factor that is expressed by mesenchymal cells and promotes processes such as mitogenesis, differentiation, and angiogenesis [2]. It mediates these functions via binding to its unique

\footnotetext{
* Correspondence: aekoch@umich.edu

${ }^{2}$ Department of Internal Medicine, 109 Zina Pitcher PI., University of Michigan Medical School, Ann Arbor, MI 48109, USA

Full list of author information is available at the end of the article
}

receptor c-Met, a receptor tyrosine kinase. c-Met is expressed by a variety of cell types, including endothelial cells (ECs) [3].

We have previously shown that HGF is elevated in the synovial fluid of patients with RA [4]. More recently, Grandaunet and colleagues [5] found that plasma levels of HGF predict the severity of joint damage in patients with RA. In the joint, we found that HGF and c-Met are elevated in the RA synovial lining compared with normal controls [4]. The report by Tsunemi and colleagues [1] supports these findings and further shows that c-Met is expressed on fibroblasts, mononuclear cells, and ECs in the RA synovium.

HGF is a heterodimeric protein composed of an $\alpha$-chain, which contains four kringle domains, and a $\beta$-chain [6]. The $\alpha$-chain binds c-Met with high affinity, whereas the $\beta$ chain is responsible for activation of c-Met. In an attempt to inhibit HGF, Date and colleagues [7] generated a cleavage product of HGF termed NK4, which contains the four kringle domains of the HGF $\alpha$-chain. Therefore, NK4 serves as an antagonist of HGF and can bind c-Met with high affinity without activating it.

As described above, one of the primary functions of HGF is to induce angiogenesis by binding to c-Met on the surface of ECs. Therefore, it was postulated that NK4 would act as a competitive inhibitor of HGF, thus inhibiting angiogenesis. Indeed, NK4 has been shown to inhibit angiogenesis in vitro and in various in vivo cancer models $[6,8,9]$. However, in addition to having antagonistic action against HGF, NK4 inhibits angiogenesis induced by vascular endothelial growth factor and basic fibroblast growth factor in a c-Met-independent fashion [9]. In addition to c-Met, NK4 binds to perlecan, a sulfate proteoglycan that interacts with the vascular endothelial basement membrane. Sakai and colleagues [9] found, specifically, that NK4 binds perlecan and prevents 
proper fibronectin assembly in the basement membrane, which inhibits several facets of angiogenesis.

These features of NK4 make it an attractive potential adjunctive therapy in angiogenic diseases. Over the past decade, numerous studies have been performed to assess the efficacy of either a recombinant NK4 protein or NK4 gene expression vector in many experimental cancer models $[3,6]$. Collectively, these studies have indicated that NK4 treatment has the potential to inhibit tumor growth, angiogenesis, and metastasis $[3,6]$. Much of the preclinical success of NK4 can be attributed to its ability to inhibit multiple pathways involved in growth and angiogenesis.

RA is driven by inflammation and angiogenesis, and thus much work has been aimed at identifying and testing potential angiogenesis inhibitors in models of experimental arthritis [10]. Tsunemi and colleagues [1] have now adopted their approach of studying the antiangiogenic properties of NK4 in cancer to experimental arthritis. Using an adenovirus vector containing the NK4 gene, they found that NK4 inhibited the development of $\beta$-glucan-induced arthritis [1]. NK4 was able to inhibit inflammation, joint swelling, and bone erosion. However, the authors did not show direct evidence of NK4 inhibiting synovial blood vessel density. Importantly, they also showed that NK4 gene therapy was effective when given therapeutically, after the onset of the experimental arthritis [1].

These results are highly encouraging in the application of NK4 as a potential adjunctive RA therapy. This report, coupled with the high expression levels of HGF and c-Met in the RA synovium, makes NK4 treatment an intriguing possibility. In the future, it will be of great interest to determine whether these effects of NK4 are observable in other animal models of RA, as not all facets of RA are represented in a singular model of the disease. Moreover, many of the effects of NK4 observed by Tsunemi and colleagues [1] are attributed to a reduction in inflammation and inflammatory cytokines. Therefore, elucidating the anti-inflammatory and antiangiogenic mechanisms of NK4 will be paramount to transitioning from an interesting candidate to a possible RA therapy.

\section{Abbreviations}

EC: Endothelial cell; HGF: Hepatocyte growth factor; RA: Rheumatoid arthritis.

\section{References}

1. Tsunemi S, Iwasaki T, Kitano S, Matsumoto K, Takagi-Kimura M, Kubo S, Tamaoki T, Sano H: Molecular targeting of hepatocyte growth factor by an antagonist, NK4, in the treatment of rheumatoid arthritis. Arthritis Res Ther 2013, 15:R75.

2. Goetsch L, Caussanel V, Corvaia N: Biological significance and targeting of c-Met tyrosine kinase receptor in cancer. Front Biosci 2013, 18:454-473.

3. You WK, McDonald DM: The hepatocyte growth factor/c-Met signaling pathway as a therapeutic target to inhibit angiogenesis. BMB Rep 2008, 41:833-839.

4. Koch AE, Halloran MM, Hosaka S, Shah MR, Haskell CJ, Baker SK, Panos RJ, Haines GK, Bennett GL, Pope RM, Ferrara N: Hepatocyte growth factor. A cytokine mediating endothelial migration in inflammatory arthritis. Arthritis Rheum 1996, 39:1566-1575.

5. Grandaunet B, Syversen SW, Hoff M, Sundan A, Haugeberg G, van Der Heijde D, Kvien TK, Standal T: Association between high plasma levels of hepatocyte growth factor and progression of radiographic damage in the joints of patients with rheumatoid arthritis. Arthritis Rheum 2011, 63:662-669.

6. Nakamura T, Sakai K, Matsumoto K: Anti-cancer approach with NK4: bivalent action and mechanisms. Anticancer Agents Med Chem 2010, 10:36-46.

7. Date K, Matsumoto K, Shimura H, Tanaka M, Nakamura T: HGF/NK4 is a specific antagonist for pleiotrophic actions of hepatocyte growth factor. FEBS Lett 1997, 420:1-6.

8. Kuba K, Matsumoto K, Date K, Shimura H, Tanaka M, Nakamura T: HGF/NK4, a four-kringle antagonist of hepatocyte growth factor, is an angiogenesis inhibitor that suppresses tumor growth and metastasis in mice. Cancer Res 2000, 60:6737-6743.

9. Sakai K, Nakamura T, Matsumoto K: Angioinhibitory action of NK4 involves impaired extracellular assembly of fibronectin mediated by perlecanNK4 association. J Biol Chem 2009, 284:22491-22499.

10. Szekanecz Z, Besenyei T, Paragh G, Koch AE: New insights in synovial angiogenesis. Joint Bone Spine 2010, 77:13-19.

10.1186/ar4320

Cite this article as: Rabquer and Koch: NK4 therapy: a new approach to target angiogenesis and inflammation in rheumatoid arthritis. Arthritis Research \& Therapy 2013, 15:119

\section{Competing interests}

The authors declare that they have no competing interests.

\section{Author details}

${ }^{1}$ Department of Biology, 611 E Porter St, Albion College, Albion, MI 49224,

USA. ${ }^{2}$ Department of Internal Medicine, 109 Zina Pitcher Pl., University of

Michigan Medical School, Ann Arbor, MI 48109, USA. 